\title{
Enlarged Controllability and Optimal Control of Sub-Diffusion Processes with Caputo Fractional Derivatives
}

\author{
Touria Karite $^{1}$, Ali Boutoulout ${ }^{1}$ and Delfim F. M. Torres ${ }^{2, *}$ \\ ${ }^{1}$ TSI Team, MACS Laboratory, Department of Mathematics \& Computer Science, Institute of Sciences, Moulay Ismail University, \\ Meknes, Morocco \\ ${ }^{2}$ Center for Research and Development in Mathematics and Applications (CIDMA), Department of Mathematics, University of Aveiro, \\ 3810-193 Aveiro, Portugal
}

Received: 4 Nov. 2018, Revised: 16 Nov. 2019, Accepted: 22 Nov. 2019

Published online: 1 Apr. 2020

\begin{abstract}
The present paper investigates the exact enlarged controllability and optimal control of a fractional diffusion equation in Caputo sense. This is done through a new definition of enlarged controllability that allows us to extend available contributions. Moreover, the problem is explored using two approaches: a reverse Hilbert uniqueness method, generalizing the approach introduced by Lions in 1988, and a penalization method, which allows us to characterize the minimum energy control.
\end{abstract}

Keywords: Fractional calculus and diffusion, Caputo derivatives and enlarged controllability, RHUM approach and minimum energy, fractional optimal control, zone and pointwise actuators.

\section{Introduction}

Calculus of fractional order began more than three centuries ago. It was first mentioned by Leibniz, in a reply to l'Hôpital, addressing the question whether the derivative remains valid for a non-integer order. The subject has been developed by several mathematicians, such as Euler, Fourier, Liouville, Grunwald, Letnikov and Riemann. Currently, other authors investigate such kind of operators and propose new fractional derivatives $[1,2,3,4,5]$. Over the last few decades, fractional calculus has gained more and more attention because of its applications in various fields of science, such as physics, engineering, economics, and biology $[6,7,8,9,10,11,12]$.

In control theory, several authors have been interested in fractional calculus since the sixties of last century. The first contributions generalize classical analytical methods and concepts for fractional order systems, such as the transfer function, frequency response, pole and zero analysis, and so on $[13,14]$. Nowadays, fractional calculus is used in the field of automatic control to obtain better and more accurate models, to develop new control strategies, and to improve the characteristics of control systems $[15,16]$.

In recent years, fractional order sub-diffusion systems have grabbed the attention of several researchers because they have outperformed the traditional integer order systems. More precisely, they can accurately characterize anomalous diffusion processes in various real-world complex systems [17,18,19,20]. In particular, fractional anomalous diffusion has been used to describe different physical scenarios, most prominently within crowded systems, for example protein diffusion within cells or diffusion through porous media. Time-fractional sub-diffusion has also been proposed as a measure of macromolecular crowding in the cytoplasm [21].

Controllability of a fractional order sub-diffusion system can be reformulated as an infinite dimensional control problem. Moreover, not all states can be reached in case of diffusion systems [22,23,24]. Because mathematical models of real systems are obtained from measures or approximation techniques, affected by perturbations if the solutions for such systems are only approximately known, control problems subject to output fractional constraints are more realistic and adapted for system analysis than the classical ones [12,25].

\footnotetext{
*Corresponding author e-mail: delfim@ua.pt
} 
Many problems in modern science are addressed with the help of optimization theory. Optimal control, as a branch of Mathematics, aims to improve the state variables of a control system in order to maximize the benefit or minimize the given cost. This is applicable to practical situations, where state variables can be temperature, a velocity field, a measure of information, etc. This is the main reason why optimal control is an attractive research area for many scientists in various disciplines. Efficient optimization and optimal control methods have been developed in order to compute the solution of fractional optimal control problems [12,26,27].

The present paper handles the controllability problem of Caputo fractional diffusion equations in presence of constraints on the state variables. This is related to the notion of enlarged controllability, which was first investigated by Lions in 1988 for hyperbolic systems [28] and later developed for linear and semilinear parabolic systems [29,30,31,32]. Moreover, we create a bridge with optimal control of systems described by fractional order differential equations. For that we prove enlarged controllability by means of a reverse Hilbert Uniqueness Method (HUM) and make use of a penalization method, which allows us to characterize the minimum energy control. We consider the Caputo fractional derivative because it allows traditional initial and boundary conditions to be included in the formulation of the problem [33]. For results associated with the Caputo-Fabrizio operators, we refer the reader to the recent paper [34].

The present paper is organized as follows. Definitions and preliminaries on fractional calculus are presented in Section 2. In Section 3, we characterize the exact enlarged controllability of the system. Section 4 is devoted to the results of the exact enlarged controllability, in two different cases: for zone and pointwise actuators. In Section 5, an optimization problem for a system of fractional order is solved using a penalization method. Sections 6 and 7 involve some examples of the two cases of actuators and conclusion. A preprint of this paper is available in [35].

\section{Preliminaries}

Let $\Omega \subset \mathbb{R}^{n}$ be bounded with a smooth boundary $\partial \Omega$. For $T>0$, denote $Q=\Omega \times[0, T]$ and $\Sigma=\partial \Omega \times[0, T]$. We consider the following abstract fractional sub-diffusion system of order $\alpha \in(0,1)$ :

$\begin{cases}{ }^{C} D^{\alpha} y(t)=\mathscr{A} y(t)+\mathscr{B} u(t), & t \in[0, T], \\ y(0)=y_{0} & \text { in } D(\mathscr{A}),\end{cases}$

where ${ }^{C} D^{\alpha}$ denotes the Caputo fractional order derivative (for details on Caputo fractional operators, see, e.g., [1, 2]). The second order operator $\mathscr{A}$ is linear and with dense domain, such that the coefficients do not depend on $t$ and generate a $C_{0}$-semi-group $(S(t))_{t \geq 0}$ on the Hilbert space $L^{2}(\Omega)$. We refer the reader to Engel and Nagel [36] as well as Renardy and Rogers [37] for properties on operator $\mathscr{A}$. In the sequel, we let $\mathscr{D}(A)$ be the domain of the operator $\mathscr{A} ; y \in L^{2}\left(0, T ; L^{2}(\Omega)\right)$ and $u \in U=L^{2}\left(0, T ; \mathbb{R}^{m}\right)$, where $m$ is the number of actuators. The initial datum $y_{0}$ is in $L^{2}(\Omega), \mathscr{B}: \mathbb{R}^{m} \longrightarrow L^{2}(\Omega)$ is the control operator, which is linear, possibly unbounded, and depending on the number and structure of actuators.

Several definitions and preliminary results are required to investigate the system (1). We begin with the most important function used in fractional calculus, i.e., Euler's gamma function, which is defined as

$$
\Gamma(n)=\int_{0}^{\infty} t^{n-1} e^{-t} d t
$$

This function is a generalization of the factorial: if $n \in \mathbb{N}$, then $\Gamma(n)=(n-1)$ !.

Definition 1(See, e.g., [1]). The left-sided Caputo fractional derivative of order $\alpha>0$ of a function $z$ is given by

$$
{ }_{0}^{C} D_{t}^{\alpha} z(t)=\left\{\begin{array}{l}
\frac{1}{\Gamma(n-\alpha)} \int_{0}^{t}(t-s)^{n-\alpha-1} \frac{d^{n}}{d s^{n}} z(s) d s, \quad n-1<\alpha<n, \quad t \geq 0, \quad n \in \mathbb{N}, \\
\frac{d^{n} z(t)}{d t^{n}}, \quad \alpha=n \in \mathbb{N} .
\end{array}\right.
$$

The right-sided is pointwise defined. The Caputo fractional derivative is a sort of regulation in the time origin for the Riemann-Liouville fractional derivative.

Definition 2(See, e.g., [38,39,40]). Let $z: \mathbb{R}^{+} \rightarrow \mathbb{R}$ be a continuous function on $\mathbb{R}^{+}$and $\alpha>0$. Then the expressions

$$
{ }_{0} I_{t}^{\alpha} z(t)=\frac{1}{\Gamma(\alpha)} \int_{0}^{t}(t-s)^{\alpha-1} z(s) d s, \quad t>0
$$

and

$$
{ }_{t} I_{T}^{\alpha} z(t)=\frac{1}{\Gamma(\alpha)} \int_{t}^{T}(s-t)^{\alpha-1} z(s) d s, \quad t<T
$$

are, respectively, called the left-sided and right-sided Riemann-Liouville integrals of order $\alpha$. 
Definition 3(See, e.g., $[38,39,40])$. Let $z: \mathbb{R}^{+} \rightarrow \mathbb{R}$. The left-sided and right-sided Riemann-Liouville fractional derivatives of order $\alpha$ are defined by

$$
{ }_{0} D_{t}^{\alpha} z(t)=\frac{1}{\Gamma(n-\alpha)} \frac{d^{n}}{d t^{n}} \int_{0}^{t}(t-s)^{n-\alpha-1} z(s) d s, \quad t>0,
$$

and

$$
{ }_{t} D_{T}^{\alpha} z(t)=\frac{1}{\Gamma(n-\alpha)}\left(-\frac{d}{d t}\right)^{n} \int_{t}^{T}(s-t)^{n-\alpha-1} z(s) d s, \quad t<T,
$$

where $\alpha \in(n-1, n), n \in \mathbb{N}$.

We always consider solutions of (1) in the weak sense. We denote that solution by $y(x, t ; u)$ and, when no possible ambiguity, we also use the short notation $y_{u}(t)$ or $y(u)$. Hence, we denote by $y_{u}(T)$ the mild solution of system (1) at the final time $T$.

Definition 4(See [41]). For $t \in[0, T]$ and any given $u \in U$, a function $y \in L^{2}\left(0, T ; L^{2}(\Omega)\right)$ is a mild solution of system (1) if it satisfies

$$
y_{u}(t)=\mathscr{R}_{\alpha}(t) y_{0}+\int_{0}^{t}(t-s)^{\alpha-1} K_{\alpha}(t-s) \mathscr{B} u(s) d s,
$$

where

$$
\mathscr{R}_{\alpha}(t)=\int_{0}^{\infty} \phi_{\alpha}(\theta) S\left(t^{\alpha} \theta\right) d \theta
$$

and

$$
K_{\alpha}(t)=\alpha \int_{0}^{\infty} \theta \phi_{\alpha}(\theta) S\left(t^{\alpha} \theta\right) d \theta
$$

with $\phi_{\alpha}(\theta)$ given by

$$
\phi_{\alpha}(\theta)=\frac{1}{\alpha} \theta^{-1-1 / \alpha} \psi_{\alpha}\left(\theta^{-1 / \alpha}\right),
$$

where $\psi_{\alpha}$ is the following probability density function:

$$
\psi_{\alpha}(\theta)=\frac{1}{\pi} \sum_{n=1}^{\infty}(-1)^{n-1} \theta^{-\alpha n-1} \frac{\Gamma(n \alpha+1)}{n !} \sin (n \pi \alpha), \quad \theta \in(0, \infty) .
$$

Remark.The probability density function (10) satisfies the following properties:

$$
\int_{0}^{\infty} e^{-\lambda \theta} \psi_{\alpha}(\theta) d \theta=e^{-\lambda^{\alpha}}, \quad \int_{0}^{\infty} \psi_{\alpha}(\theta) d \theta=1, \quad \alpha \in(0,1)
$$

and

$$
\int_{0}^{\infty} \theta^{v} \phi_{\alpha}(\theta) d \theta=\frac{\Gamma(1+v)}{\Gamma(1+\alpha v)}, \quad v \geq 0 .
$$

Let $H: L^{2}\left(0, T ; \mathbb{R}^{m}\right) \rightarrow L^{2}(\Omega)$ be defined as

$$
H u=\int_{0}^{T}(T-s)^{\alpha-1} K_{\alpha}(T-s) \mathscr{B} u(s) d s, \quad \forall u \in L^{2}\left(0, T ; \mathbb{R}^{m}\right),
$$

where $m$ is the number of actuators. We assume that $\left(S^{*}(t)\right)_{t \geq 0}$ is a strongly continuous semi-group generated by $A^{*}$ on the state space $L^{2}(\Omega)$. For $v \in L^{2}(\Omega)$, one has

$$
\begin{aligned}
\langle H u, v\rangle & =\left\langle\int_{0}^{T}(T-s)^{\alpha-1} K_{\alpha}(T-s) \mathscr{B} u(s) d s, v\right\rangle_{L^{2}(\Omega)} \\
& =\int_{0}^{T}\left\langle(T-s)^{\alpha-1} K_{\alpha}(T-s) \mathscr{B} u(s), v\right\rangle_{L^{2}(\Omega)} d s \\
& =\int_{0}^{T}\left\langle u(s), \mathscr{B}^{*}(T-s)^{\alpha-1} K_{\alpha}^{*}(T-s) v\right\rangle_{L^{2}\left(0, T ; \mathbb{R}^{m}\right)} d s \\
& =\left\langle u, H^{*} v\right\rangle,
\end{aligned}
$$


where by $\langle\cdot, \cdot\rangle$, we denote the duality pairing of space $L^{2}(\Omega), \mathscr{B}^{*}$ is the adjoint operator of $\mathscr{B}$, and

$$
K_{\alpha}^{*}(t)=\alpha \int_{0}^{\infty} \theta \phi_{\alpha}(\theta) S^{*}\left(t^{\alpha} \theta\right) d \theta .
$$

In order to prove our main results, the following lemmas are needed.

Lemma 1(See [42]). Let the reflection operator $\mathscr{Q}$ on the interval $[0, T]$ be defined as follows:

$$
\mathscr{Q} h(t):=h(T-t)
$$

for function $h$ that is differentiable and integrable. Then, the following relations hold:

$$
\mathscr{Q}_{0} I_{t}^{\alpha} h(t)={ }_{t} I_{T}^{\alpha} \mathscr{Q} h(t), \quad \mathscr{Q}_{0} D_{t}^{\alpha} h(t)={ }_{t} D_{T}^{\alpha} \mathscr{Q} h(t)
$$

and

$$
{ }_{0} I_{t}^{\alpha} \mathscr{Q} h(t)=\mathscr{Q}_{t} I_{T}^{\alpha} h(t), \quad{ }_{0} D_{t}^{\alpha} \mathscr{Q} h(t)=\mathscr{Q}_{t} D_{T}^{\alpha} h(t) .
$$

Lemma 2(See, e.g., [43]). For $t \in[a, b]$ and $n-1<\alpha<n, n \in \mathbb{N}$, the following integration by parts formula holds:

$$
\int_{a}^{b} f(t)_{0}^{C} D_{t}^{\alpha} g(t) d t=\sum_{r=0}^{k-1}(-1)^{k-1-r}\left[g^{r}(t)_{t} D_{b}^{\alpha-1-r} f(t)\right]_{t=a}^{t=b}+(-1)^{k} \int_{a}^{b} g(t)_{t} D_{b}^{\alpha} f(t) d t .
$$

In particular, if $0<\alpha<1$, then

$$
\int_{a}^{b} f(t)_{0}^{C} D_{t}^{\alpha} g(t) d t=\left[g(t)_{t} I_{b}^{1-\alpha} f(t)\right]_{t=a}^{t=b}+\int_{a}^{b} g(t)_{t} D_{b}^{\alpha} f(t) d t .
$$

We also recall the fractional Green's formula:

Lemma 3(See, e.g., $[39,44])$. Let $0<\alpha \leq 1$ and $t \in[0, T]$. Then,

$$
\begin{aligned}
& \int_{0}^{T} \int_{\Omega}\left({ }_{0}^{C} D_{t}^{\alpha} y(x, t)+\mathscr{A} y(x, t)\right) \varphi(x, t) d x d t=\int_{0}^{T} \int_{\Omega} y(x, t)\left({ }_{t} D_{T}^{\alpha} \varphi(x, t)+\mathscr{A}^{*} \varphi(x, t)\right) \\
& +\int_{0}^{T^{T}} \int_{\partial \Omega} y(x, t)_{t} I_{T}^{1-\alpha} \varphi(x, t) d \Gamma d t-\int_{0}^{T} \int_{\partial \Omega} y(x, t) \frac{\partial \varphi(x, t)}{\partial v_{\mathscr{A}}}+\int_{0}^{T} \int_{\partial \Omega} \frac{\partial y(x, t)}{\partial v_{\mathscr{A}}} \varphi(x, t) d \Gamma d t
\end{aligned}
$$

for any $\varphi \in C^{\infty}(\bar{Q})$.

\section{Regional enlarged controllability}

We extend the definition of controllability first introduced by Lions in [45] to the case of sub-diffusion fractional systems. For that, we consider a nonempty sub-vectorial space $G \subset L^{2}(\Omega)$, which is supposed to be closed and convex.

Definition 5.Given a final time $T>0$, we say that system (1) is exactly enlarged controllable (i.e., G-controllable) if, for every $y_{0}$ in a suitable functional space, there exists a control $u$ such that

$$
y(\cdot, T ; u) \in G .
$$

Remark.Obviously, the notion of exact enlarged controllability depends on $G$.

Remark.If $G=\{0\}$, then we get the classical concept of exact controllability from Definition 5 .

Remark.Exact controllability implies exact enlarged controllability (EEC) for every set $G$. The inverse is, however, untrue.

Theorem 1.System (1) is said to be exactly enlarged controllable if, and only if,

$$
G-\left\{\mathscr{R}_{\alpha}(T) y_{0}\right\} \cap \operatorname{ImH} \neq \emptyset .
$$


Proof. Suppose that one has exact enlarged controllability (EEC) of (1) relatively to $G$, which means that $y_{u}(T) \in G$. Then, $y_{u}(T)=\mathscr{R}_{\alpha}(T) y_{0}+H u$ and, denoting

$$
w=y_{u}(T)-\mathscr{R}_{\alpha}(T) y_{0}=H u,
$$

it follows that $w \in \operatorname{Im} H$ and $w \in G-\left\{\mathscr{R}_{\alpha}(T) y_{0}\right\}$. Thus, (16) holds. Conversely, suppose (16) is true. Then, there exists $z \in G-\left\{\mathscr{R}_{\alpha}(T) y_{0}\right\}$ such that $z \in \operatorname{ImH}$. So, there exists $u \in L^{2}\left(0, T ; \mathbb{R}^{m}\right)$ such that $z=H u$. Hence, $z=H u \in G\left\{\mathscr{R}_{\alpha}(T) y_{0}\right\}$, $H u+\mathscr{R}_{\alpha}(T) y_{0}=y_{u}(T) \in G$, and we have EEC relatively to $G$.

We recall that an actuator is defined by a couple $(D, f)$, where $D$ is a nonempty closed part of $\bar{\Omega}$, which represents the geometric support of the actuator, and $f \in L^{2}(D)$, which defines the spacial distribution of the action on the support $D$. In the case of a pointwise actuator, $D=\{b\}$ and $f=\delta(b-\cdot)$, where $\delta$ is the Dirac mass concentrated in $b$. For more details on actuators, we refer the interested reader to $[46,47]$.

Definition 6.The actuator $(D, f)$ is said to be G-strategic if one has exact enlarged controllability relatively to $G$.

\section{Extended RHUM approach}

Now, we generalize the RHUM introduced by Lions in $[48,49]$ to the fractional-order case. The aim is to find the control steering system (1) from the initial state $y_{0}$ into the functional subspace $G$. Let us denote by $G^{\circ}$ the polar space of $G$. Hence,

$$
\varphi_{0} \in G^{\circ} \Longleftrightarrow\left\langle\varphi_{0}, \phi\right\rangle=0 \quad \forall \phi \in G,
$$

where $\langle\cdot, \cdot\rangle$ denotes the scalar product in $L^{2}(\Omega)$. Let us also denote by $\mathscr{A}^{*}$ the adjoint operator of $\mathscr{A}$ and, for any $\varphi_{0} \in G^{\circ}$, consider the following adjoint system:

$$
\left\{\begin{array}{l}
{ }_{t} D_{T}^{\alpha} Q \varphi(t)=-\mathscr{A}^{*} Q \varphi(t), \\
\lim _{t \rightarrow T^{-}}{ }_{t} I_{T}^{1-\alpha} Q \varphi(t)=\varphi_{0} \in D\left(\mathscr{A}^{*}\right) \subseteq L^{2}(\Omega) .
\end{array}\right.
$$

From Lemma 2, (17) can be rewritten as follows:

$$
\left\{\begin{array}{l}
{ }_{0} D_{t}^{\alpha} \varphi(t)=-\mathscr{A}^{*} \varphi(t), \\
\lim _{t \rightarrow 0^{+}} I_{t}^{1-\alpha} \varphi(t)=\varphi_{0} \in D\left(\mathscr{A}^{*}\right) \subseteq L^{2}(\Omega),
\end{array}\right.
$$

with solution given by $\varphi(t)=-t^{\alpha-1} K_{\alpha}^{*}(t) \varphi_{0}$.

\subsection{Excitation of the system with a zone actuator}

We consider system (1) excited by a zone actuator $\mathscr{B} u(t)=\chi_{D} f(x) u(t)$. Then, the system is written as follows:

$$
\left\{\begin{array}{l}
{ }_{0}^{C} D_{t}^{\alpha} y(t)=\mathscr{A} y(t)+\chi_{D} f(x) u(t), \quad t \in[0, T], \\
y(0)=y_{0} \in D(\mathscr{A}) .
\end{array}\right.
$$

Let $w_{i}(x)$ denote the eigenfunctions of operator $\mathscr{A}$ associated with the eigenvalues $\lambda_{i}$. For any $\varphi_{0} \in G^{\circ}$, we define the following semi-norm on $G^{\circ}$ :

$$
\left\|\varphi_{0}\right\|_{G^{\circ}}^{2}:=\int_{0}^{T}\langle f, \varphi(t)\rangle_{L^{2}(D)}^{2} d t
$$

Theorem 2.The semi-norm (20) defines a norm on $G^{\circ}$ if $\left\langle w_{i}, f\right\rangle_{L^{2}(D)} \neq 0$. In that case, we have exact enlarged controllability relatively to $G$.

Proof. We consider the following problem:

$$
\left\{\begin{array}{l}
{ }_{0}^{C} D_{t}^{\alpha} \Psi(t)=\mathscr{A} \Psi(t)+\chi_{D} f(x) u(t), \quad t \in[0, T] \\
\Psi(0)=y_{0} \in D(\mathscr{A})
\end{array}\right.
$$


The solution $\Psi:[0, T] \rightarrow L^{2}(\Omega)$ of (21) is continuous. If we can find $\varphi_{0} \in G^{\circ}$ such that

$$
\Psi(T) \in G
$$

then $u=\langle f, \varphi(t)\rangle_{L^{2}(D)}$ is the control that ensures the exact enlarged controllability relatively to $G$ and

$$
y(u)=\Psi .
$$

To explain (22), it is necessary to introduce the orthogonal projection $\mathscr{P}$ on the orthogonal of $G$, denoted by $G^{\perp}$. Let us define the affine operator $M: G^{\circ} \rightarrow G^{\perp}$ such that

$$
M \varphi_{0}=\mathscr{P}(\Psi(T))
$$

Then, we need to solve equation

$$
M \varphi_{0}=0
$$

For that, we decompose $M$ into two parts: a linear and a constant one. Let $\Psi_{0}$ be solution of

$$
\left\{\begin{array}{l}
{ }_{0}^{C} D_{t}^{\alpha} \Psi_{0}(t)=\mathscr{A} \Psi_{0}(t)+\chi_{D} f(x) u(t), \quad t \in[0, T] \\
\Psi_{0}(0)=0
\end{array}\right.
$$

and $\Psi_{1}$ solution of

$$
\left\{\begin{array}{l}
{ }_{0}^{C} D_{t}^{\alpha} \Psi_{1}(t)=\mathscr{A} \Psi_{1}(t), \quad t \in[0, T] \\
\Psi_{1}(0)=y_{0} \in D(\mathscr{A})
\end{array}\right.
$$

Then,

$$
M \varphi_{0}=\mathscr{P}\left(\Psi_{0}(T)\right)+\mathscr{P}\left(\Psi_{1}(T)\right)
$$

where we set $M_{0} \varphi_{0}=\mathscr{P}\left(\Psi_{0}(T)\right)$ with $M_{0} \in \mathscr{L}\left(G^{\circ}, G^{\perp}\right)$. From (24) and (27), we can solve

$$
M_{0} \varphi_{0}=-\mathscr{P}\left(\Psi_{1}(T)\right) .
$$

For that, we compute the scalar product

$$
\mu=\left\langle M_{0} \varphi_{0}, \varphi_{0}\right\rangle, \quad \varphi_{0} \in G^{\circ},
$$

where $\langle\cdot, \cdot\rangle$ is the dual pairing of $G^{\perp}$ and $G^{\circ}$. By definition,

$$
\langle\mathscr{P}(\tilde{g}), \bar{g}\rangle=0, \quad \forall \bar{g} \in G^{\circ} .
$$

Using (30), we have

$$
\mu=\left\langle\Psi_{0}, \varphi_{0}\right\rangle
$$

for $\bar{g}=\varphi_{0}, \tilde{g}=\Psi_{0}(T)$. To compute the last expression (31), we multiply system (25) by $\varphi$, integrating over $Q=\Omega \times[0, T]$. We obtain that

$$
\int_{0}^{T} \int_{\Omega}{ }_{0}^{C} D_{t}^{\alpha} \Psi_{0}(t) \varphi(t) d x d t-\int_{0}^{T} \int_{\Omega} \mathscr{A} \Psi_{0}(t) \varphi(t) d x d t=\int_{0}^{T} \int_{\Omega} \chi_{D} f(x) u(t) \varphi(t) d x d t .
$$

Using Lemma 3 (fractional Green's formula), we have

$$
\begin{aligned}
-\int_{0}^{T} \int_{\Omega} \mathscr{A} \Psi_{0}(t) \varphi(t) d x d t=-\int_{0}^{T} \int_{\partial \Omega} \frac{\partial \Psi_{0}(t)}{\partial v_{\mathscr{A}}} \varphi(t) d \sigma d t & \\
& +\int_{0}^{T} \int_{\partial \Omega} \Psi_{0}(t) \frac{\partial \varphi(t)}{\partial v_{\mathscr{A}}} d \sigma d t-\int_{0}^{T} \int_{\Omega} \Psi_{0} \mathscr{A}^{*} \varphi(t) d x d t
\end{aligned}
$$

and

$$
\begin{aligned}
\int_{0}^{T} \int_{\Omega}{ }_{0}^{C} D_{t}^{\alpha} \Psi_{0}(t) \varphi(t) d x d t & =\int_{0}^{T} \int_{\Omega} \Psi_{0}(t)_{0}^{C} D_{t}^{\alpha} \varphi(t) d x d t+\int_{\partial \Omega} \Psi_{0}(T) \lim _{t \rightarrow T} I_{T}^{1-\alpha} \varphi(T) d \sigma \\
& -\int_{\partial \Omega} \Psi_{0}(0) \lim _{t \rightarrow T} I_{T}^{1-\alpha} \varphi(0) d \sigma .
\end{aligned}
$$


From (32) and (33), it follows that

$$
\left\langle M_{0} \varphi_{0}, \varphi_{0}\right\rangle=\int_{0}^{T}\left(\langle f(x), \varphi(t)\rangle_{L^{2}(D)}\right)^{2} d t=\left\|\varphi_{0}\right\|_{G^{\circ}}^{2} .
$$

Hence,

$$
\mu=\int_{0}^{T}\left(\langle f(x), \varphi(t)\rangle_{L^{2}(D)}\right)^{2} d t
$$

The essential point now is that the previous formula (34) is a semi-norm on $G^{\circ}$. We prove that if $\left\langle w_{i}, f\right\rangle_{L^{2}(D)} \neq 0$, then the mapping (20) is a norm, which is equivalent to the norm of $G^{\circ}$. The mapping (20) is a norm on $G^{\circ}$ :

$$
\left\|\varphi_{0}\right\|_{G^{\circ}}=0 \Longleftrightarrow\langle f, \varphi(t)\rangle_{L^{2}(D)}^{2}=0
$$

which is equivalent to

$$
-\sum_{i=1}^{\infty} t^{\alpha-1} \alpha \int_{0}^{\infty} \theta \phi_{\alpha}(\theta) e^{\lambda_{i}\left(t^{\alpha} \theta\right)} d \theta\left\langle f, w_{i}\right\rangle\left\langle\varphi_{0}, w_{i}\right\rangle=0
$$

Thus, (35) gives

$$
\left\langle f, w_{i}\right\rangle\left\langle\varphi_{0}, w_{i}\right\rangle=0
$$

Using the assumption that $\left\langle f, w_{i}\right\rangle \neq 0$, we deduce that $\left\langle\varphi_{0}, w_{i}\right\rangle=0$. Therefore, $\varphi_{0}=0$, (20) defines a norm on $G^{\circ}$, and $\mu$ is an isomorphism from $G^{\circ}$ to $G^{\perp}$. Moreover, equation (28) admits a unique solution.

\subsection{Excitation of the system with a pointwise actuator}

Now, we consider system (1) excited by a pointwise actuator. In this case, the control is of type $B u(t)=\delta(x-b) u(t)$, where $b \in \Omega$ refers to the location of the actuator and $u \in U$. Hence, system (1) is written as follows:

$$
\left\{\begin{array}{l}
{ }_{0}^{C} D_{t}^{\alpha} y(t)=\mathscr{A} y(t)+\delta(x-b) u(t), \quad t \in[0, T] \\
y(0)=y_{0} \in D(\mathscr{A})
\end{array}\right.
$$

For $\varphi_{0} \in G^{\circ}$, we consider the adjoint system

$$
\left\{\begin{array}{l}
{ }_{t} D_{T}^{\alpha} Q \varphi(t)=-\mathscr{A}^{*} Q \varphi(t), \\
\lim _{t \rightarrow T^{-}}{ }_{t} I_{T}^{1-\alpha} Q \varphi(t)=\varphi_{0} \in D\left(\mathscr{A}^{*}\right) \subseteq L^{2}(\Omega),
\end{array}\right.
$$

and the mapping

$$
\left\|\varphi_{0}\right\|_{G^{\circ}}^{2}:=\int_{0}^{T} \varphi^{2}(b, t) d t
$$

which defines a semi-norm on $G^{\circ}$. Let us consider system

$$
\left\{\begin{array}{l}
{ }_{0}^{C} D_{t}^{\alpha} \Phi(t)=\mathscr{A} \Phi(t)+\delta(x-b) u(t), \quad t \in[0, T] \\
\Phi(0)=y_{0} \in D(\mathscr{A})
\end{array}\right.
$$

and the operator $M: G^{\circ} \rightarrow G^{\perp}$ defined by

$$
M \varphi_{0}=\mathscr{P}(\Phi(T))
$$

where we write $M$ as

$$
M \varphi_{0}=\mathscr{P}\left(\Phi_{0}(T)+\Phi_{1}(T)\right)
$$

with $\Phi_{0}$ and $\Phi_{1}$ solutions of systems

$$
\left\{\begin{array}{l}
{ }_{0}^{C} D_{t}^{\alpha} \Phi_{0}(t)=\mathscr{A} \Phi_{0}(t)+\delta(x-b) u(t), \quad t \in[0, T] \\
\Phi_{0}(0)=0
\end{array}\right.
$$


and

$$
\left\{\begin{array}{l}
{ }_{0}^{C} D_{t}^{\alpha} \Phi_{1}(t)=\mathscr{A} \Phi_{1}(t), \quad t \in[0, T] \\
\Phi_{1}(0)=y_{0} \in D(\mathscr{A})
\end{array}\right.
$$

respectively. Let us set $M_{0} \varphi_{0}=\mathscr{P}\left(\Phi_{0}(T)\right)$ with $M_{0} \in \mathscr{L}\left(G^{\circ}, G^{\perp}\right)$. Then, all reduces to solve

$$
M_{0} \varphi_{0}=-\mathscr{P}\left(\Phi_{1}(T)\right)
$$

Similar arguments as the ones used in Section 4.1 allow us to prove the following result:

Theorem 3.If $w_{i}(b) \neq 0$, then the mapping (38) defines a norm on $G^{\circ}$ and one has exact enlarged controllability (EEC) relatively to G. Moreover, the control

$$
u=\varphi(b, t)
$$

ensures the EEC into $G$.

\section{Fractional optimal control}

In this section, we are concerned with the following optimization problem:

$$
\left\{\begin{array}{l}
\inf \mathscr{J}(u) \\
u \in U_{a d}
\end{array}\right.
$$

where

$$
\mathscr{J}(u)=\frac{1}{2} \int_{0}^{T}\|u\|_{U}^{2} d t
$$

and the feasible set $U_{a d}=\left\{u \in U \mid y_{u}(T) \in G\right\}$ is assumed to be non-empty.

Theorem 4.Assume that one has exact enlarged controllability relatively to $G$. Then, the optimal control problem (43) has a unique solution given by $u^{*}(t)=\langle f, \varphi(t)\rangle$ in case of a zone actuator, and $u^{*}(t)=\varphi(b, t)$ in case of a pointwise actuator. Such control ensures the transfer of system (1) into $G$ with a minimum energy cost, in the sense of $\mathscr{J}$.

Proof.Suppose that we have exact enlarged controllability relatively to $G$. Then, we set $\varepsilon>0$ and we consider the following problem:

$$
\mathscr{J}_{\varepsilon}(u, z)=\frac{1}{2} \int_{0}^{T} u^{2}(t) d t+\frac{1}{2 \varepsilon} \int_{Q}\left({ }_{0}^{C} D_{t}^{\alpha} z(t)-\mathscr{A} z(t)-\chi_{D} f(x) u(t)\right)^{2} d Q
$$

where

$$
\left\{\begin{array}{l}
{ }_{0}^{C} D_{t}^{\alpha} z(t)-\mathscr{A} z(t)-\chi_{D} f(x) u(t) \in L^{2}(Q), \\
z(0)=z_{0} \in D(\mathscr{A}), \\
z_{u}(T) \in G .
\end{array}\right.
$$

The set of pairs $(u, z)$ that verify (45), denoted by $W$, is nonempty, and we consider problem

$$
\left\{\begin{array}{l}
\inf \mathscr{J}_{\varepsilon}(u, z) \\
(u, z) \in W
\end{array}\right.
$$

Let $\left\{u_{\varepsilon}, z_{\varepsilon}\right\}$ be solution of (46). Then,

$$
0<\mathscr{J}_{\varepsilon}\left(u_{\varepsilon}, z_{\varepsilon}\right)=\inf \mathscr{J}_{\varepsilon}(u, z)<\inf \mathscr{J}_{\varepsilon}(u)<\infty, \quad u \in U_{a d},
$$

where $\mathscr{J}_{\varepsilon}(u)=\frac{1}{2} \int_{0}^{T} u^{2}(t) d t$. Tending $\varepsilon$ to 0 , we conclude that

$\left\{\begin{array}{l}\left\|u_{\varepsilon}\right\| \leq C \\ \left\|_{0}^{C} D_{t}^{\alpha} z(x, t)-\mathscr{A} z(x, t)-\chi_{D} f(x) u(t)\right\| \leq C \sqrt{\varepsilon}\end{array}\right.$ 
where $C$ represents different positive constants independent of $\varepsilon$. It follows from (48) that

$$
\left\|{ }_{0}^{C} D_{t}^{\alpha} z(x, t)-\mathscr{A} z(x, t)\right\| \leq C(1+\sqrt{\varepsilon}) .
$$

Hence, when $\varepsilon \rightarrow 0$, we have that $u_{\varepsilon}$ is bounded and we can extract a sequence such that

$$
\begin{aligned}
& u_{\varepsilon} \rightarrow \tilde{u} \quad \text { weakly in } U, \\
& z_{\varepsilon} \rightarrow z \text { weakly in } L^{2}(Q) .
\end{aligned}
$$

By the semi-continuity of $\mathscr{J}$, one has

$$
\mathscr{J}\left(u^{*}\right) \leq \liminf \mathscr{J}_{\varepsilon}\left(u_{\varepsilon}\right) \leq \liminf \mathscr{J}_{\varepsilon}\left(u_{\varepsilon}, z_{\varepsilon}\right) .
$$

Therefore,

$$
\mathscr{J}\left(u^{*}\right)=\inf \mathscr{J}(u), \quad u \in U_{a d}
$$

and

$$
u^{*}=\tilde{u}
$$

Define

$$
p_{\varepsilon}=-\frac{1}{\varepsilon}\left({ }_{0}^{C} D_{t}^{\alpha} z_{\varepsilon}(x, t)-\mathscr{A} z_{\varepsilon}(x, t)-\chi_{D} f(x) u_{\varepsilon}(t)\right) .
$$

The Euler equation relatively to problem (46) is given by

$$
\int_{0}^{T} u_{\mathcal{\varepsilon}}(t) u(t) d t-\int_{0}^{T}\left\langle p_{\mathcal{\varepsilon}},{ }_{0}^{C} D_{t}^{\alpha} \eta(t)-\mathscr{A} \eta(t)\right\rangle d t=\int_{0}^{T}\left\langle p_{\varepsilon}, f\right\rangle u(t) d t
$$

with $u \in U_{a d}$ and $\eta$ such that

$$
\begin{cases}{ }_{0}^{C} D_{t}^{\alpha} \eta(t)-\mathscr{A} \eta(t)=\chi_{D} f(x) u(t) & \text { in } \quad Q, \\ \eta(0)=0 & \text { on } \Omega \\ \eta(T) \in G & \end{cases}
$$

We deduce that $p_{\varepsilon}$ satisfies

$$
\begin{cases}{ }_{0}^{C} D_{t}^{\alpha} p_{\varepsilon}(t)-\mathscr{A} p_{\varepsilon}(t)=\chi_{D} f(x)\left\langle p_{\varepsilon}, f\right\rangle_{L^{2}(D)} & \text { in } Q, \\ p_{\varepsilon}(0)=0 & \text { on } \Omega,\end{cases}
$$

and $\left\langle\eta(T), p_{\varepsilon}(T)\right\rangle=0$ for all $\eta$ with $\eta(T) \in G$. Then, $p_{\varepsilon} \in G^{\circ}$. If we suppose that

$$
\int_{0}^{T}\left\langle p_{\varepsilon}, f\right\rangle^{2} d t \geq C\left\|p_{\varepsilon}(T)\right\|_{H_{0}^{1}(\Omega)}^{2},
$$

then we can switch to the limit when $\varepsilon$ tends to 0 . Moreover, because we have exact enlarge controllability relatively to $G$, we obtain the following optimality problem:

$$
\begin{cases}{ }_{0}^{C} D_{t}^{\alpha} z(t)-\mathscr{A} z(t)=\chi_{D} f(x) u(t) & \text { in } Q, \\ z(0)=z_{0}(x) & \text { on } \Omega, \\ { }_{0}^{C} D_{t}^{\alpha} p(t)-\mathscr{A} p(t)=\chi_{D} f(x)\langle p, f\rangle_{L^{2}(D)} & \text { in } Q, \\ p(0)=0 & \text { on } \Omega, \\ p(T) \in G^{\circ} . & \end{cases}
$$

Thus, we take $p(T) \in G^{\circ}$ and we introduce the solution $\varphi$ of (18). Then, $\psi=z$ if $\psi(T) \in G$, which proves that (28) has a unique solution for $\varphi_{0} \in G^{\circ}$. 


\section{Examples}

Two examples illustrate the obtained results as follows:

\subsection{Example 1: case of a zonal actuator}

Let us consider the following time fractional differential equation with a zonal actuator: $B u(t)=\chi_{[a, b]} u(t), 0 \leq a \leq b \leq 1$,

$$
\begin{cases}{ }_{0}^{C} D_{t}^{0.4} z(t)=\Delta z(t)+\chi_{[a, b]} u(t) & {[0,1] \times[0, T],} \\ z(x, 0)=z_{0}(x) & {[0,1],} \\ z(0, t)=z(1, t)=0 & {[0, T] .}\end{cases}
$$

Here, the state space is $L^{2}(0,1)$. Since the operator $\mathscr{A}=\Delta=\frac{\partial^{2}}{\partial x^{2}}$ generates a compact, analytic, self-adjoint $C_{0}$-semigroup, we have $\mathscr{A}=\Delta=\frac{\partial^{2}}{\partial x^{2}}$ and

$$
S(t) z(x)=\sum_{i=1}^{+\infty} e^{\lambda_{i} t}\left(z, w_{i}\right)_{L^{2}(0,1)} w_{i}(x),
$$

where $\lambda_{i}=-i^{2} \pi^{2}$ and $w_{i}(x)=\sqrt{2} \sin (i \pi x)$. Moreover,

$$
\begin{aligned}
K_{0.4}(t) z(x) & =0.4 \int_{0}^{\infty} \theta \phi_{0.4}(\theta) S\left(t^{0.4} \theta\right) z d \theta \\
& =0.4 \int_{0}^{\infty} \theta \phi_{0.4}(\theta) \sum_{i=1}^{\infty} e^{\lambda_{i} t^{0.4} \theta}\left(z, w_{i}\right)_{L^{2}(0,1)} w_{i}(x) d \theta \\
& =0.4 \sum_{i=1}^{\infty}\left(z, w_{i}\right)_{L^{2}(0,1)} w_{i}(x) \int_{0}^{\infty} \theta \phi_{0.4}(\theta) e^{\lambda_{i} t^{0.4}} \theta d \theta .
\end{aligned}
$$

It follows from (12) and Taylor's expansion of the exponential that

$$
\begin{aligned}
K_{0.4}(t) z(x) & =0.4 \sum_{i=1}^{\infty}\left(z, w_{i}\right)_{L^{2}(0,1)} w_{i}(x) \sum_{j=0}^{\infty} \int_{0}^{\infty} \frac{\left(\lambda_{i} t^{0.4}\right)^{j}}{j !} \theta^{j+1} \phi_{0.4}(\theta) d \theta \\
& =\sum_{i=1}^{\infty}\left(z, w_{i}\right)_{L^{2}(0,1)} w_{i}(x) \sum_{j=0}^{\infty} \frac{0.4(j+1)\left(\lambda_{i} t^{0.4}\right)^{j}}{\Gamma(1+0.4 j+0.4)} \\
& =\sum_{i=1}^{\infty} E_{0.4,0.4}\left(\lambda_{i} t^{0.4}\right)\left(z, w_{i}\right)_{L^{2}(0,1)} w_{i}(x)
\end{aligned}
$$

where $E_{p, q}(z):=\sum_{i=0} \frac{z^{i}}{\Gamma(p i+q)}, \operatorname{Re}(p)>0, q, z \in \mathbb{C}$, is the generalized Mittag-Leffler function (see [50]). Similarly, we have:

$$
\begin{aligned}
\mathscr{R}_{0.4}(t) z(x) & =\int_{0}^{\infty} \phi_{0.4}(\theta) S\left(t^{0.4} \theta\right) z d \theta \\
& =\sum_{i=0}^{\infty}\left(z, w_{i}\right)_{L^{2}(0,1)} E_{0.4,1}\left(\lambda_{i} t^{0.4}\right) w_{i}(x) .
\end{aligned}
$$

Since operator $\Delta$ generates a compact, analytic, self-adjoint and continuous semigroup, it follows that

$$
\begin{aligned}
\left(H^{*} z\right)(t) & =\mathscr{B}^{*}(T-t)^{-0.6} K_{0.4}^{*}(T-t) z(t) \\
& =\mathscr{B}^{*}(T-t)^{-0.6} \sum_{i=1}^{\infty} E_{0.4,0.4}\left(\lambda_{i}(T-t)^{0.4}\right)\left(z, w_{i}\right)_{L^{2}(0,1)} w_{I}(x) \\
& =(T-t)^{-0.6} \sum_{i=1}^{\infty} E_{0.4,0.4}\left(\lambda_{i}(T-t)^{0.4}\right)\left(z, w_{i}\right)_{L^{2}(0,1)} \int_{a}^{b} w_{i}(x) d x . \\
& =(T-t)^{-0.6} \sum_{i=1}^{\infty} E_{0.4,0.4}\left(\lambda_{i}(T-t)^{0.4}\right)\left(z, w_{i}\right)_{L^{2}(0,1)} \frac{\sqrt{2}}{i \pi}[\cos (i \pi x)]_{a}^{b} \\
& =(T-t)^{-0.6} \sum_{i=1}^{\infty} E_{0.4,0.4}\left(\lambda_{i}(T-t)^{0.4}\right)\left(z, w_{i}\right)_{L^{2}(0,1)} \frac{\sqrt{2}}{i \pi} \sin \frac{i \pi(a+b)}{2} \sin \frac{i \pi(a-b)}{2} .
\end{aligned}
$$


Moreover, by Theorem 2, we get that if system (49) is enlarged controllable, then

$$
\begin{aligned}
\varphi_{0} \rightarrow\left\|\varphi_{0}\right\|_{\left(L^{2}(0,1)\right)^{*}} & =\sum_{i=1}^{\infty} t^{-0.6} 0.4 \int_{0}^{\infty} \theta \phi_{0.4}(\theta) e^{\lambda_{i}\left(t^{0.4} \theta\right)} d \theta\left\langle f, w_{i}\right\rangle\left\langle\varphi_{0}, w_{i}\right\rangle \\
& =t^{-0.6} K_{0.4}(t)\left\langle\varphi_{0}, w_{i}\right\rangle \\
& =t^{-0.6} \sum_{i=1}^{\infty} E_{0.4,0.4}\left(\lambda_{i} t^{0.4}\right)\left(z, w_{i}\right)_{L^{2}(0,1)} w_{i}(x)\left\langle\varphi_{0}, w_{i}\right\rangle
\end{aligned}
$$

defines a norm on $\left(L^{2}(0,1)\right)^{*}$. We find that the control given by

$$
u^{*}(t)=t^{-0.6} \sum_{i=0}^{+\infty} E_{0.4,0.4}\left(\lambda_{i} t^{0.4}\right)\left(z, w_{i}\right)_{L^{2}(0,1)}\left\langle\varphi_{0}, w_{i}\right\rangle
$$

steers system (49) to $L^{2}(0,1)$ at time $T$.

\subsection{Example 2: case of a pointwise actuator}

We now consider the following system with a pointwise control $B u(t)=u(t) \delta(x-b), 0<b<1$ :

$$
\begin{cases}{ }_{0}^{C} D_{t}^{0.4} z(t)=\Delta z(t)+u(t) \delta(x-b) & {[0,1] \times[0, T],} \\ z(x, 0)=z_{0}(x)=0 & {[0,1],} \\ z(0, t)=z(1, t)=0 & {[0, T] .}\end{cases}
$$

Let the position of the actuator be $b=1 / 3$. Similar to the first example, we have:

$$
\begin{aligned}
& \lambda_{i}=-i^{2} \pi^{2}, \quad w_{i}(x)=\sqrt{2} \sin (i \pi x), \quad x \in[0,1], \\
& S(t) z(x)=\sum_{i=1}^{+\infty} e^{\lambda_{i} t}\left(z, w_{i}\right)_{L^{2}(0,1)} w_{i}(x), \\
& \text { and } K_{0.4}(t) z(x)=\sum_{i=1}^{+\infty} E_{0.4,0.4}\left(\lambda_{i} t^{0.4}\right)\left(z, w_{i}\right)_{L^{2}(0,1)} w_{i}(x) .
\end{aligned}
$$

Moreover, by Theorem 3, we get that if system (50) is enlarged controllable, then

$$
\begin{aligned}
\varphi_{0} \rightarrow\left\|\varphi_{0}\right\|_{\left(L^{2}(0,1)\right)^{*}} & =\int_{0}^{T}\left\|(T-s)^{-0.6} K_{0.4}^{*}(T-s) \varphi_{0}(b)\right\|^{2} d s \\
& =\int_{0}^{T}\left\|(T-s)^{-0.6} \sum_{i=0}^{+\infty} E_{0.4,0.4}\left(\lambda_{i}(T-s)^{0.4}\right)\left(z, w_{i}\right)_{L^{2}(0,1)} \varphi_{0}(b)\right\|^{2} d s
\end{aligned}
$$

defines a norm on $\left(L^{2}(0,1)\right)^{*}$. We also have that $M \varphi_{0}=\mathscr{P}\left(\varphi_{1}(T)\right)$ is an affine operator from $\left(L^{2}(0,1)\right)^{*}$ to $\left(L^{2}(0,1)\right)$, where $\varphi_{1}(T)$ is the solution of system

$$
\left\{\begin{array}{l}
{ }_{0}^{C} D_{t}^{0.4} \varphi_{1}(t)=\Delta \varphi_{1}(t)+(T-t)^{-0.6} K_{0.4}^{*}(T-t) \varphi_{0}(b) \\
\varphi_{1}(0)=0 \\
\varphi_{1}(0, t)=\varphi_{1}(1, t)=0
\end{array}\right.
$$

Then, by Theorem 4, we find that the control given by

$$
u^{*}(t)=(T-t)^{-0.6} \sum_{i=0}^{+\infty} E_{0.4,0.4}\left(\lambda_{i}(T-s)^{0.4}\right)\left(z, w_{i}\right)_{L^{2}(0,1)} \varphi_{0}(b)
$$

steers system (50) to $L^{2}(0,1)$ at time $T$, where $\varphi_{0}$ is the solution of

$$
M_{0} \varphi_{0}=-\mathscr{P}\left(\Phi_{1}(T)\right)
$$

and $\Phi_{1}(t)$ solves

$$
\left\{\begin{array}{l}
{ }_{0}^{C} D_{t}^{0.4} \Phi_{1}(t)=\Delta \Phi_{1}(t), \quad t \in[0, T] \\
\Phi_{1}(0)=z_{0}(x)=0 \in D(\mathscr{A}) \\
\Phi_{1}(0, t)=\Phi_{1}(1, t)=0
\end{array}\right.
$$

Moreover, $u^{*}$ is the solution of the minimum problem (43). 


\section{Conclusion}

The present paper addressed fractional diffusion equations in the sense of Caputo. We investigated exact enlarged controllability for such control systems using an extended Reverse Hilbert Uniqueness Method (RHUM) and a penalization technique covering both zone and pointwise actuators. The optimal control of a minimum energy problem has been explicitly characterized. The two methods complete each other: using the RHUM approach, we computed the control steering the system for both cases of zone and pointwise actuators; and using the penalization method, we proved that such control is unique. We claim that our techniques and results can be adapted to cover boundary conditions of Dirichlet, Neumann or mixed type, and to deal with other classes of controls (e.g., distributed controls). The present results can be extended to more recent notions of derivatives, e.g., to Atangana-Baleanu operators [51,52]. Another research will conduct numerical experiments illustrating theoretical results.

\section{Acknowledgements}

The present work was supported by Hassan II Academy of Science and Technology (Project N 630/2016), Morocco, and by FCT through the Center for Research and Development in Mathematics and Applications (CIDMA), project with references UIDB/04106/2020 and UIDP/04106/2020, Portugal. The authors are strongly grateful to the anonymous referees for their suggestions and invaluable comments.

\section{References}

[1] I. Podlubny, Fractional differential equations, Mathematics in Science and Engineering, 198, Academic Press, Inc., San Diego, CA, 1999.

[2] A. A. Kilbas, H. M. Srivastava and J. J. Trujillo, Theory and applications of fractional differential equations, North-Holland Mathematics Studies, 204, Elsevier Science B. V., Amsterdam, 2006.

[3] A. B. Malinowska and D. F. M. Torres, Introduction to the fractional calculus of variations, Imperial College Press, London, 2012.

[4] A. B. Malinowska, T. Odzijewicz and D. F. M. Torres, Advanced methods in the fractional calculus of variations, Springer Briefs in Applied Sciences and Technology, Springer, Cham, 2015.

[5] D. Baleanu, K. Diethelm, E. Scalas and J. J. Trujillo, Fractional calculus models and numerical methods, Series on Complexity, Nonlinearity and Chaos, 5, World Scientific Publishing Co. Pte. Ltd., Hackensack, NJ, 2016.

[6] M. Ichise, Y. Nagayanagi and T. Kojima, An analog simulation of non integer order transfer functions for analysis of electrode processes, J. Elect. Chem. 13, 253-265 (1971).

[7] J. A. Tenreiro Machado, I. S. Jesus, A. Galhano and J. Boaventura Cunha, Fractional order electromagnetics, Signal Proc. 86, 2637-2644 (2006).

[8] S. Owyed, M. A. Abdou, A. Abdel-Aty, A. A. Ibraheem, R. Nekhili, D. Baleanu, New optical soliton solutions of space-time fractional nonlinear dynamics of microtubules via three integration schemes, Journal of Intelligent and Fuzzy systems 38, 28592866 (2020).

[9] J. L. Battaglia, O. Cois, L. Puigsegur and A. Oustaloup, Solving an inverse heat conduction problem using a non-integer identified model, Int. J. Heat Mass Transf. 44(14), 2671-2680 (2001).

[10] R. L. Bagley and P. J. Torvik, A theoretical basis for the application of fractional calculus to viscoelasticity, J. Rheol. 27(3), 201-210 (1983).

[11] G. Catania and S. Sorrentino, Analytical modelling and experimental identification of viscoelastic mechanical systems, in Advances in fractional calculus, 403-416, Springer, Dordrecht, 2007.

[12] R. P. Agarwal, D. Baleanu, J. J. Nieto, D. F. M. Torres and Y. Zhou, A survey on fuzzy fractional differential and optimal control nonlocal evolution equations, J. Comput. Appl. Math. 339, 3-29 (2018).

[13] A. Oustaloup, Systèmes asservis linéaires d'ordre fractionnaire: théorie et pratique, Edition Masson, 1983.

[14] M. Axtell and M. E. Bise, Fractional calculus applications in control systems, Proc. IEEE Nat. Aerosp. Elect., New-York, 563-566, 1990.

[15] A. Debbouche and D. F. M. Torres, Approximate controllability of fractional nonlocal delay semilinear systems in Hilbert spaces, Int. J. Contr. 86(9), 1577-1585 (2013).

[16] M. D. Ortigueira, Fractional calculus for scientists and engineers, Lecture Notes in Electrical Engineering, 84, Springer, Dordrecht, 2011.

[17] R. Metzler and J. Klafter, The random walk's guide to anomalous diffusion: a fractional dynamics approach, Phys. Rep. 339(1), $77 \mathrm{pp}(2000)$.

[18] M. Weiss, M. Elsner, F. Kartberg and T. Nilsson, Anomalous sub-diffusion is a measure for cytoplasmic crowding in living cells, Biophys. J. 87(5), 3518-3524 (2004).

[19] W. Chen, H. G. Sun, X. Zhang and D. Korošak, Anomalous diffusion modeling by fractal and fractional derivatives, Comput. Math. Appl. 59(5), 1754-1758 (2010). 
[20] Y. Luchko, Fractional calculus models for the anomalous diffusion processes and their analysis, Application of Mathematics in Technical and Natural Sciences, AIP Conf. Proc. 1301 (1), 623-635 (2010).

[21] F. Ge, Y. Q. Chen and C. Kou, Regional analysis of time-fractional diffusion processes, Springer, Cham, 2018.

[22] A. El Jaï and A. J. Pritchard, Sensors and controls in the analysis of distributed systems, Ellis Horwood Ltd., Chichester, 1988.

[23] F. Ge, Y. Q. Chen and C. Kou, Regional gradient controllability of sub-diffusion processes, J. Math. Anal. Appl. 440(2), 865-884 (2016).

[24] F. Ge, Y. Q. Chen and C. Kou, Regional boundary controllability of time fractional diffusion processes, IMA J. Math. Contr. Infor. 34(3), 871-888 (2017).

[25] A. Debbouche, J. J. Nieto and D. F. M. Torres, Optimal solutions to relaxation in multiple control problems of Sobolev type with nonlocal nonlinear fractional differential equations, J. Opt. Theor. Appl. 174(1), 7-31 (2017).

[26] A. Flores-Tlacuahuac and L. T. Biegler, Optimization of fractional order dynamic chemical processing systems, Ind. Eng. Chem. Res. 53, 5110-5127 (2014).

[27] Y. Zhou and E. Casas, Fractional systems and optimization, Optimization 63(8), 1153-1156 (2014).

[28] J. L. Lions, Contrôlabilité exacte, perturbations et stabilisation de systèmes distribués. Tome 1, Recherches en Mathématiques Appliquées, 8, Masson, Paris, 1988.

[29] El H. Zerrik and F. Ghafrani, Minimum energy control subject to output constraints numerical approach, IEE Proc. Contr. Theor. Appl. 149(1), 105-110 (2002).

[30] T. Karite and A. Boutoulout, Regional constrained controllability for parabolic semilinear systems, Int. J. Pure Appl. Math. 113(1), $113-129$ (2017).

[31] T. Karite and A. Boutoulout, Regional boundary controllability of semi-linear parabolic systems with state constraints, Int. J. Dyn. Syst. Differ. Equ. 8(1-2), 150-159 (2018).

[32] T. Karite, A. Boutoulout and D. F. M. Torres, Enlarged controllability of Riemann-Liouville fractional differential equations, $J$. Comput. Nonlin. Dynam. 13(9), 090907 (2018).

[33] D. N. Susahab and S. Shahmorad, Application of the fractional differential transform method to fractional-order integro-differential equations with nonlocal boundary conditions, J. Comput. Appl. Math. 234(3), 883-891 (2010).

[34] T. Karite, A. Boutoulout and A. Khan, Minimum energy control of fractional linear systems using Caputo-Fabrizio derivative. In: Recent Advances in Modeling, Analysis and Systems Control: Theoretical Aspects and Applications, E. H. Zerrik et al. (eds.), Springer, Cham, 2020.

[35] T. Karite, A. Boutoulout and D. F. M. Torres, Enlarged controllability and optimal control of sub-diffusion processes with Caputo fractional derivatives, preprint, arXiv:1911.10199 [math.OC].

[36] K. J. Engel and R. Nagel, A short course on operator semigroups, Universitext, Springer, New York, 2006.

[37] M. Renardy and R. C. Rogers, An introduction to partial differential equations, second edition, Texts in Applied Mathematics, 13, Springer-Verlag, New York, 2004.

[38] O. P. Agrawal, A general formulation and solution scheme for fractional optimal control problems, Nonlinear Dynam. 38(1-4), 323-337 (2014).

[39] G. M. Bahaa, Fractional optimal control problem for differential system with control constraints, Filomat, 30(8), 2177-2189 (2016).

[40] G. M. Mophou, Optimal control of fractional diffusion equation, Comput. Math. Appl. 61(1), 68-78 (2011).

[41] F. Mainardi, P. Paradisi and R. Gorenflo, Probability distributions generated by fractional diffusion equations, FRACALMO preprint (2007), and arXiv:0704.0320v1.

[42] M. Klimek, On solutions of linear fractional differential equations of a variational type, Czestochowa University of Technology, Czestochowa, 2009.

[43] I. Podlubny and Y. Q. Chen, Adjoint fractional differential expressions and operators. Proc. ASME 2007 International Design Engineering Technical Conferences \& Computers and Information in Engineering Conference IDETC/CIE 2007, September 4-7, 2007, Las Vegas, Nevada, USA.

[44] G. M. Bahaa and Q. Tang, Optimality conditions of fractional diffusion equations with weak Caputo derivatives and variational formulation, J. Fract. Calc. Appl. 9(1), 100-119 (2018).

[45] J. L. Lions, Contrôlabilité exacte, perturbations et stabilisation de systèmes distribués. Tome 2, Recherches en Mathématiques Appliquées, 9, Masson, Paris, 1988.

[46] A. El Jaï and A. J. Pritchard, Capteurs et actionneurs dans l'analyse des systèmes distribués, Recherches en Mathématiques Appliquées, 3, Masson, Paris, 1986.

[47] El H. Zerrik, A. El Jai and A. Boutoulout, Actuators and regional boundary controllability of parabolic system, Int. J. Syst. Sci. 31(1), 73-82 (2000).

[48] J. L. Lions, Optimal control of systems governed by partial differential equations, Translated from the French by S. K. Mitter. Die Grundlehren der Mathematischen Wissenschaften, Band 170, Springer-Verlag, New York, 1971.

[49] J. L. Lions, Exact controllability, stabilization and perturbations for distributed systems, SIAM Rev. 30(1), 1-68 (1988).

[50] R. Gorenflo, A. A. Kilbas, F. Mainardi and S. V. Rogosin, Mittag-Leffler functions, related topics and applications, Springer Monographs in Mathematics, Springer, Heidelberg, 2014.

[51] A. Atangana and D. Baleanu, New fractional derivatives with nonlocal and non-singular kernel: theory and application to heat transfer model, Therm. Sci. 20(2), 763-769 (2016).

[52] S. Kumar and P. Pandey, Quasi wavelet numerical approach of non-linear reaction diffusion and integro reaction-diffusion equation with Atangana-Baleanu time fractional derivative, Chaos Solit. Fract. 130, 109456 (2020). 